\title{
Cyclin D1 and cyclin D-dependent kinases enhance oral keratinocyte proliferation but do not block keratinocyte differentiation
}

\author{
MICHAEL WOODS ${ }^{1}$, RIMA PANT ${ }^{2}$ and SANJAY M. MALLYA ${ }^{1}$ \\ ${ }^{1}$ School of Dentistry, University of California, Los Angeles, 10833 Le Conte Avenue, Los Angeles, CA 90095; \\ ${ }^{2}$ University of Connecticut School of Dental Medicine, 263 Farmington Avenue, Farmington, CT 06030, USA
}

Received June 9, 2010; Accepted July 9, 2010

DOI: 10.3892/ijo_00000799

\begin{abstract}
Maintenance of oral epithelial homeostasis requires a fine balance between cell proliferation and differentiation. However, the molecular mechanisms that couple these processes, and its deregulation in tumorigenesis are not fully understood. Cyclin D1 and its kinase partners CDK4 and CDK6 play an important role in regulating the G1-S phase of the cell cycle. Deregulation of cyclin D1 is a frequent event in oral squamous cell carcinoma. Here, we examined whether overexpression of cyclin D1, CDK4 and CDK6 can deregulate the link between oral keratinocyte proliferation and differentiation. Our results show that cyclin D1 and its kinase partners CDK4 and CDK6 enhance keratinocyte proliferation, but are not sufficient to block calcium-induced keratinocyte differentiation and suggest that deregulation of these G1-regulatory kinases alone is insufficient to uncouple the link between proliferation and differentiation
\end{abstract}

\section{Introduction}

The stratified squamous epithelium of the oral mucosa is composed predominantly of tightly packed keratinocytes with varying degrees of differentiation. The deepest layer consists of least differentiated basal cells. As basal cells move toward the surface, they exit the cell cycle and start to undergo differentiation (1). A fine balance between cell proliferation and differentiation is crucial for maintenance of epithelial homeostasis and is deregulated in cancer. However, the molecular mechanisms that underlie the coupling between proliferation and differentiation are not well understood.

It is well established that cyclin D1 plays a key role in the G0/G1-S phase of the cell cycle (2). Cyclin D1 binds to and

Correspondence to: Dr Sanjay M. Mallya, Division of Diagnostic and Surgical Sciences, CHS 53-068, UCLA School of Dentistry, Los Angeles, CA 90095-1668, USA

E-mail: smallya@dentistry.ucla.edu

Key words: cyclin D1, cyclin D-dependent kinases, oral keratinocytes activates its cyclin-dependent kinase partners CDK4/6, effecting phosphorylation and inactivation of the retinoblastoma protein. Cyclin D1 is a key oncogene that plays an important role in several human cancers, including oral squamous cell carcinoma (SCC). Deregulation of cyclin D1, either by genomic amplification or by overexpression, is a frequent event in oral squamous cell carcinoma $(3,4)$. Transgenic mice with overexpression of cyclin D1 in the oroesophageal epithelium develop epithelial dysplasia (5) and manifest increased susceptibility to carcinogen-induced neoplasia (6), underscoring cyclin D1's role as a driver oncogene for this tumor type. However, the precise mechanisms by which cyclin D1 exerts its oncogenic effects and the contributions of its kinase partners, CDK4 and CDK6, to this process are not fully understood.

Given its importance in cell cycle control and its frequent deregulation in cancer, the cyclin D1 pathway is a potential candidate that may be the crucial link between proliferation and differentiation. Indeed, recent evidence shows that downregulation of cyclin D1 is important for proper initiation of keratinocyte differentiation (7). Furthermore, overexpression of CDK6 is known to block differentiation in mouse astrocytes $(8,9)$. Similar roles for CDK6 in blocking cell differentiation have also been described for murine erythyroid cells (10), osteoclasts (11) and osteoblasts (12). Thus, regulation of cyclin D1 and/or its kinases CDK4/6 may be an essential step that links cell cycle exit and initiation of differentiation. Here, we examined whether overexpression of cyclin D1 or its kinase partners CDK4 and CDK6 was sufficient to uncouple the link between oral keratinocyte proliferation and differentiation.

\section{Materials and methods}

Cells and culture conditions. OKF6-TERT1 and OKF6-TERT2 cells (obtained from Dr James Rheinwald, Brigham and Women's Hospital, Boston, MA) are immortalized, but nontransformed oral keratinocyte cell lines that stably express hTERT-the catalytic subunit of telomerase (13). Cells were grown in keratinocyte serum-free medium (Invitrogen Corp., Carlsbad, CA) supplemented with bovine pituitary extract $(25 \mu \mathrm{g} / \mathrm{ml})$, epidermal growth factor $(0.2 \mathrm{ng} / \mathrm{ml})$, penicillin $(100 \mathrm{IU} / \mathrm{ml})$ and streptomycin $(100 \mu \mathrm{g} / \mathrm{ml})$. The calcium 
concentration of the media was adjusted to $0.03 \mathrm{mM}$. To induce keratinocyte differentiation, the calcium concentration of the growth media was increased to $1.2 \mathrm{mM}$.

Lentivirus constructs and viral transductions. Plasmids containing human $C D K 4$ and $C D K 6$ cDNA sequences (14) were obtained from the plasmid repository Addgene (Addgene plasmids 1874 and 1866, respectively). A plasmid containing the human cyclin D1 cDNA was kindly provided by Dr Andrew Arnold, University of Connecticut Health Center, Farmington, CT. The cDNA sequences were excised from the parental plasmid vectors and ligated into the multiple cloning site of the lentiviral expression vector pCDH1-MCS1EF1-copGFP (pCDH1; System Biosciences, Mountain View, CA). Pseudoviral particles were produced in 293TN cells using the packaging plasmid pPACKH1-GAG, pPACKH1-REV (System Biosciences), and the envelope plasmid pVSV-G (15) following an established protocol (16). Viral particles were concentrated and resuspended in keratinocyte serum-free medium and used to transduce OKF6-TERT1 and OKF6TERT2 cells.

Cell growth and cell cycle assays. Cells were seeded into 24-well plates at densities of 20,000 cells per well. At 24, 48 and $72 \mathrm{~h}$, the media were aspirated and the plates were stored at $-80^{\circ} \mathrm{C}$. Cell proliferation was assayed using the CyQuant nucleic acid fluorescence assay kit, according to the manufacturer's instructions (Invitrogen). For analysis of cell cycle distribution, exponentially growing cells were centrifuged at $300 \mathrm{x} \mathrm{g}$ for $5 \mathrm{~min}$. The cell pellet was resuspended in complete growth media containing $2 \mu \mathrm{g} / \mathrm{ml}$ of Hoechst 33342 DNA staining reagent (Invitrogen). DNA content was analyzed on a FACScan flow cytometer (Beckton-Dickinson, Franklin Lakes, NJ).

Immunoblotting. Cells were lysed in radioimmunoprecipitation assay (RIPA) buffer by gentle rocking for $15 \mathrm{~min}$ at $4^{\circ} \mathrm{C}$. Adherent cells were removed with a cell scraper and the resulting lysates were incubated for $1 \mathrm{~h}$ on ice. Cell debris was removed by centrifugation at $10,000 \times \mathrm{g}$ for $10 \mathrm{~min}$ at $4^{\circ} \mathrm{C}$. Equal amounts of protein were layered onto reducing SDS-PAGE gels and electroblotted onto polyvinylidene fluoride microporous membranes. The membranes were incubated in blocking buffer $(20 \mathrm{mM}$ Tris, $500 \mathrm{mM} \mathrm{NaCl}$, $1 \%$ casein) for $1 \mathrm{~h}$ and subsequently incubated overnight at $4^{\circ} \mathrm{C}$ with primary antibodies. The primary antibodies used were as follows: mouse monoclonal anti-human cyclin D1 (clone DCS-6), mouse monoclonal anti-human CDK4 (DCS-35), rabbit polyclonal anti-human CDK6 (C-21, Santa Cruz Biotechnology, Santa Cruz, CA), involucrin (clone SY-5, SigmaAldrich, St. Louis, MO) and mouse monoclonal antibody against human GAPDH, as loading control (Abcam Inc., Cambridge, MA). Binding of the primary antibody was detected using an appropriate horseradish peroxidaseconjugated IgG (Santa Cruz Biotechnology) and Luminol reagent (Santa Cruz Biotechnology) and subsequent exposure to X-ray film or imaged using a CCD camera.

Immunofluorescence. Cells were allowed to attach overnight to glass coverslips in 12-well plates. The calcium concentration of the media was adjusted to $1.2 \mathrm{mM}$ and the cells were incubated for $48 \mathrm{~h}$. Cells were fixed with $4 \%$ formaldehyde for $10 \mathrm{~min}$ and washed in PBS. To detect E-cadherin, cells were blocked with $5 \%$ goat serum and incubated with the primary antibody (rabbit monoclonal anti-human E-Cadherin, 24E10, Cell Signaling Technology, Beverley, MA) for $2 \mathrm{~h}$ at room temperature. Bound primary antibody was detected using Alexa Fluor 546-conjugated goat anti-rabbit IgG (Invitrogen) and the nuclei were counterstained with TOPO-3 (Invitrogen).

Tumor samples and immunohistochemistry. De-identified formalin-fixed, paraffin-embedded tissues from human oral SCC tumors were obtained from the Oral Pathology Biopsy service at the University of Connecticut School of Dental Medicine and from the Co-operative Human Tissue Network. Studies were approved by the institutional review board at the University of Connecticut Health Center.

Paraffin-embedded tissues were sectioned onto glass slides. Sections were deparaffinized in xylene, cleared through a graded ethanol series and then hydrated in distilled water. For epitope retrieval, slides were heated for $15 \mathrm{~min}$ in citrate buffer, $\mathrm{pH}$ 6.0, in an electric pressure cooker. Endogenous peroxidase was quenched with $3 \% \mathrm{H}_{2} \mathrm{O}_{2}$ and the sections were incubated for $30 \mathrm{~min}$ in $5 \%$ normal serum to reduce nonspecific binding. Sections were incubated at room temperature with antibodies to the following proteins: CDK4 and CDK6 (Santa Cruz Biotechnology; 1:200 for $60 \mathrm{~min}$ ) and Ki67 (Novocastra Laboratories Newcastle upon Tyne, UK). Binding of the primary antibodies was detected using the appropriate biotinylated $\mathrm{IgG}$ and the avidin-biotin complex (Vector Laboratories, Burlingame, CA) using 3,3-diaminobenzidine as a chromagen (Invitrogen). Sections were counterstained in hematoxylin.

Immunohistochemical staining was scored subjectively on a 5-point scale based on the numbers of cells stained in three discrete nests of tumor cells $(0,<10 \% ; 1,10-25 \% ; 2$, $25-50 \% ; 3,50-75 \% ; 4,>75 \%)$. Tumors with a score of 3 or 4 were considered to have overexpression.

\section{Results}

Overexpression of cyclin D1,CDK4 and CDK6 enhances oral keratinocyte proliferation. Using lentiviral vectors we generated oral keratinocyte cell lines that stably overexpress cyclin CDK4, CDK6 and cyclin D1. All transduced cell lines showed robust overexpression of the target proteins (Fig. 1). Given their role in the G1-S phase regulation, we examined the effects of cyclin D1 and its kinase partners on keratinocyte proliferation. We first examined cell proliferation using the CyQuant cell proliferation assay. Compared with empty vector transduced cells both cyclin D-dependent kinases and cyclin D1 significantly increased cell proliferation of in OKF6-TERT1 cells (Fig. 2). There was no significant difference in the degree of enhancement of cell proliferation caused by overexpression of cyclin D1 or its partner kinases. Flow cytometry analyses revealed that overexpression of cyclin D1, CDK4 and CDK6 all resulted in a redistribution of the cell-cycle, with an $\sim 50 \%$ increase in cells in S-phase compared with empty vector transduced cells (Fig. 3). For all 


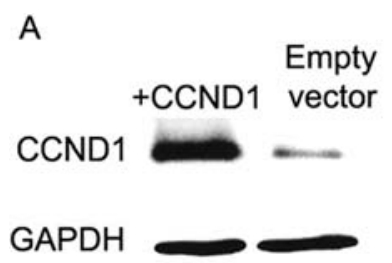

B

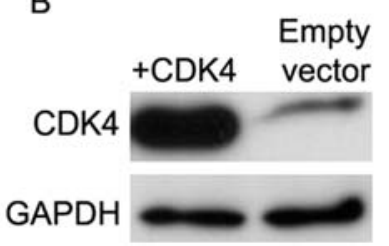

C

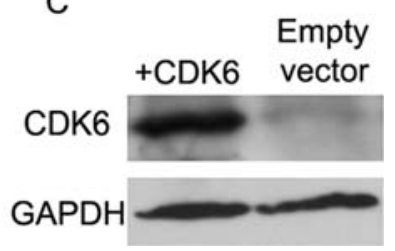

Figure 1. Stable transduction of OKF6-TERT1 cells with (A) Cyclin D1, (B) CDK4 and (C) CDK6. Whole cell lysates from transduced cells were immunoblotted to detect expression of the target protein. Expression was compared with endogenous protein levels in cells transduced with the empty vector.

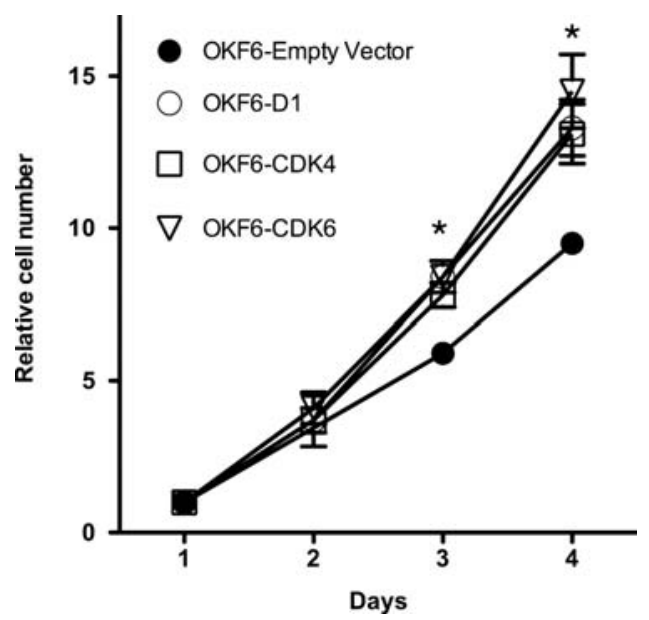

Figure 2. Analysis of cell proliferation in OKF6-TERT1 cells overexpressing cyclin D1, CDK4 and CDK6, determined using the CyQuant cell proliferation assay. ${ }^{*} \mathrm{p} \leq 0.05$, compared with empty vector transduced cells.

three cell lines this difference was significant, compared with empty vector transduced cells $(\mathrm{p} \leq 0.001)$. Similar results were observed when cyclin D1, CDK4 or CDK6 were overexpressed in another keratinocyte cell line, OKF6-TERT2 (data not shown).

Overexpression of cyclin D1,CDK4 and CDK6 do not block oral keratinocyte differentiation. Previous studies have suggested that cyclin D1 downregulation is important for keratinocyte differentiation. Also, CDK6 overexpression blocks differentiation in a variety of cell types $(8,10,11)$. Thus, we examined whether overexpression of cyclin D1 or its dependent kinases was sufficient to block keratinocyte differentiation. To induce differentiation, keratinocytes were grown in the presence of a high concentration of calcium, a known stimulus for keratinocyte differentiation (17). Within $4 \mathrm{~h}$ after increasing the calcium concentration in the media, empty vector transduced OKF6-TERT1 cells started to change shape and made intercellular contacts. After $72 \mathrm{~h}$ following stimulation of differentiation, OKF6-TERT1 cells demonstrated an increase in the expression of involucrin, a marker of keratinocyte differentiation. This increase in involucrin expression was not blocked by overexpression of either cyclin D1 or its kinases CDK4 or CDK6 (Fig. 4A). We also examined the expression of E-cadherin, another marker of keratinocyte differentiation. In the proliferative state, Ecadherin was not detected in any of the cell lines (Fig. 4B). Upon calcium-induced differentiation, empty vector transduced cells showed membranous expression of E-cadherin, along the sites of intercellular contacts. This differentiation-induced response was not altered by overexpression of either cyclin D1 or CDK4/CDK6 kinases (Fig. 4B).

Increased CDK4 and CDK6 expression in human oral SCC. Overexpression of cyclin D1 is a frequent event in oral SCC. However, the contribution of its partner kinases CDK4 and CDK6 to the development of oral SCC is less well studied. Thus, we examined the expression of CDK4 and CDK6 in human oral SCC. In normal oral epithelium, we observed strong nuclear expression of CDK4 and CDK6 limited to the basal cell layer. Of the 25 oral SCCs examined, $68 \%$ of the tumors showed increased expression of either CDK4 or CDK6 or both kinases. CDK4 expression was increased in 11 (44\%) and CDK6 in 13 (52\%) of the tumors, respectively (Fig. 5). Overexpression of the kinases was not mutually
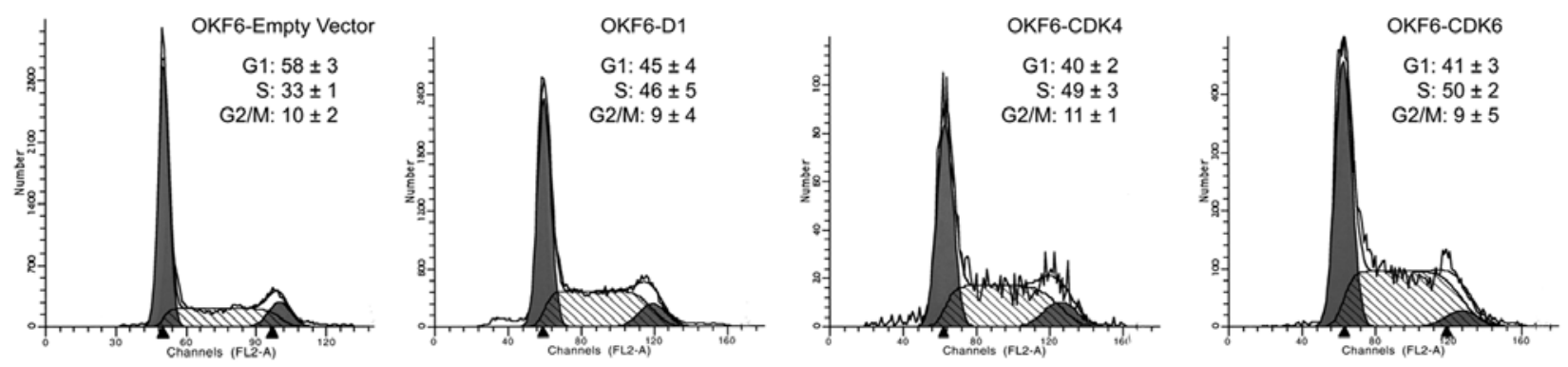

Figure 3. Cell cycle distribution in OKF6-TERT1 cells overexpressing cyclin D1, CDK4 and CDK6. Proliferating cells were stained with Hoechst 33342 and the DNA content was analyzed by flow cytometry. 
A

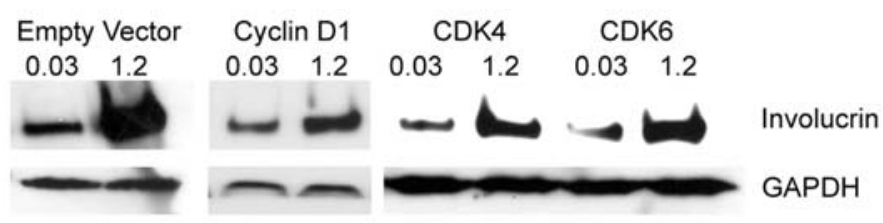

B

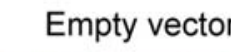

Cyclin D1

CDK4 CDK6

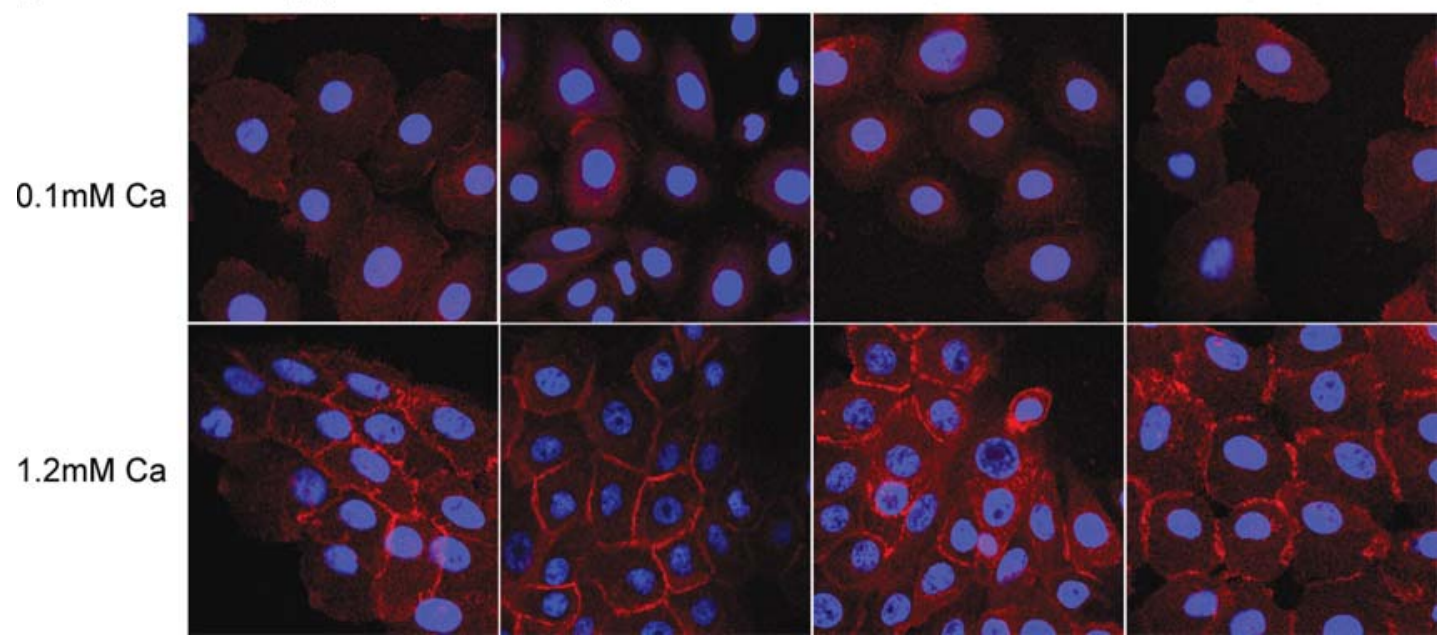

Figure 4. Expression of differentiation markers in OKF6-TERT1 cells overexpressing cyclin D1, CDK4 and CDK6. (A) Cells were treated with $1.2 \mathrm{mM}$ calcium for $72 \mathrm{~h}$ and whole cell lysates were immunoblotted to detect expression of involucrin. Expression was compared with involucrin protein levels in cells transduced with the empty vector. Expression of GAPDH was used as a loading control. (B) Cells were grown on coverslips and treated with $1.2 \mathrm{mM}$ calcium for $48 \mathrm{~h}$. Expression of E-cadherin was determined using immunofluorescence.

\section{A: CDK4}

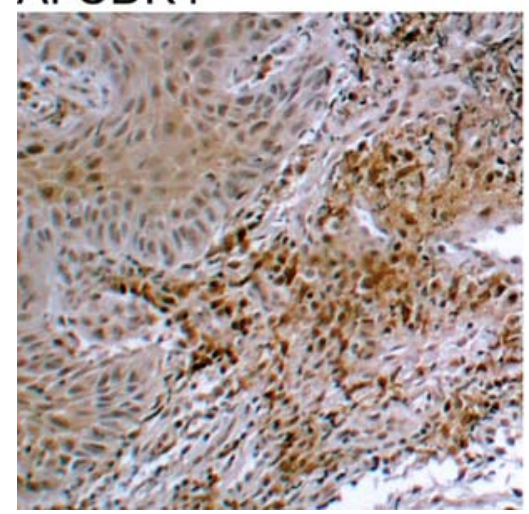

B: CDK6

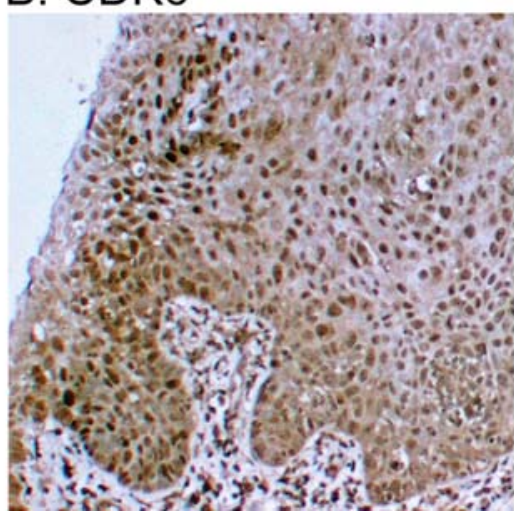

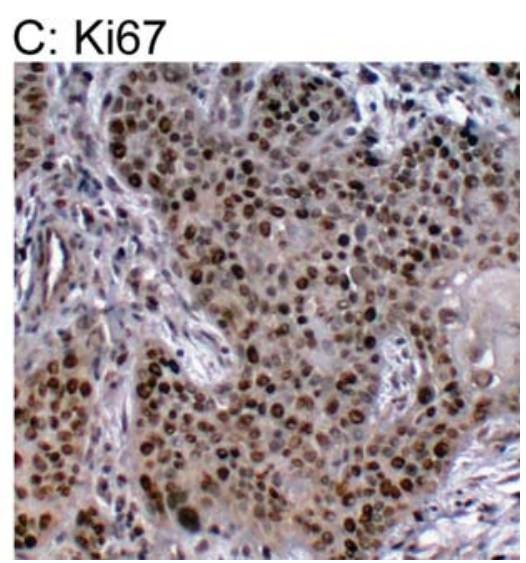

Figure 5. Expression of CDK4 and CDK6 and Ki67 in human oral SCC. Sections from human oral SCC were analyzed by immunohistochemistry for expression of CDK4, CDK6 and Ki67. Representative examples of immunostaining are shown.

exclusive, expression of both kinases was noted in 7 (28\%) cases. There was no correlation between expression of CDK4 and CDK6. Overall, there was a tendency for tumors with high expression of CDK4/CDK6 to have increased expression of the proliferation marker Ki67.

\section{Discussion}

Maintenance of oral epithelial homeostasis requires precise temporal and spatial regulation of cell proliferation and cell differentiation. The balance between proliferation and differentiation is deregulated in cancer, many cancers exhibit both loss of normal growth control and an immature cellular phenotype resulting from a block of differentiation (18). Cyclin D1, a key regulator of the G1-S phase of the cell cycle, is a well established oncogene that is important for development of oral SCC. Cyclin D1's effects on cell proliferation are thought to underlie its oncogenic effect. However, it is not known whether cyclin D1 and the kinases that mediate its functions are essential to regulate the link between proliferation and differentiation. Given its important role in regulating the G1-S transition, the cyclin D1 pathway is an attractive candidate that may potentially mediate a coupling between these two processes. Recent data show that 
cyclin D1 downregulation is important for induction of differentiation of human epidermal keratinocytes (7). Thus, we examined whether overexpression of cyclin D1 or its kinase partners CDK4/6 could block calcium-induced differentiation of oral keratinocytes. In this study we demonstrate that oral keratinocytes with forced expression of cyclin D1, CDK4 or CDK6 manifested significantly enhanced proliferation. This is consistent with the well-established role of cyclin D1 in the G1-S transition. However, despite the deregulated proliferation, oral keratinocytes that overexpressed cyclin D1, CDK4 or CDK6 responded to calcium-induced differentiation, as evidenced by morphological changes as well as expression of two differentiation markers - involucrin and E-cadherin. These findings suggest that overexpression of cyclin D1, CDK4 or CDK6 alone is not sufficient to block differentiation of oral keratinocytes. Nevertheless, our data do not completely rule out a role for cyclin D1 and its partner kinases in regulating the link between proliferation and differentiation. Perhaps, uncoupling these processes may require deregulation of additional pathways. For example, dermal keratinocytes that overexpress CDK4 and p21, in addition to cyclin D1 lack terminal differentiation (19). Our data are in contrast with those from other cell types where CDK6 overexpression blocks differentiation (8-12). This may possibly reflect differences in the tissue specific effects of the cyclin D1-dependent kinases.

Our findings are in contrast with a previous report that primary murine dermal keratinocytes with induced Cyclin D1 transgene expression are resistant to calcium-induced terminal differentiation (20). Possibly, this discrepancy may reflect a disparity between oral and dermal keratinocytes. It is also important to consider that in the previous study, the resistance to differentiation was assayed by observation of morphological changes, whereas in our study we also assayed changes in molecular markers of keratinocyte differentiation.

Interestingly, cyclin D1-dependent kinases alone were sufficient to enhance cell proliferation, suggesting that the amount of cyclin D may not be limiting the functional activation of these kinases. This emphasizes the contribution of the kinases to oral keratinocyte pathophysiology. Indeed, our finding that CDK4 and CDK6 are overexpressed in human oral SCCs further emphasizes the importance of the contribution of both these kinases to human oral neoplasia. There is evidence that cyclin D1 may have effects that are independent of its cdk-mediated actions $(21,22)$. However, the contributions of these cdk-independent functions to cyclin D1's oncogenic role are not fully understood. Our finding that CDK4 and CDK6 were capable of enhancing proliferation suggests that cyclin D1's proliferative effects are mediated for most part, via activation of its kinase partners, at least in oral keratinocytes.

In conclusion, our findings suggest that cyclin D1 and the cyclin D-dependent kinases are sufficient to enhance proliferation. However, when overexpressed alone, they are insufficient to block keratinocyte differentiation.

\section{Acknowledgements}

This work was supported by grants DE014773 and DE007302 from the National Institutes of Health.

\section{References}

1. Presland RB and Jurevic RJ: Making sense of the epithelial barrier: what molecular biology and genetics tell us about the functions of oral mucosal and epidermal tissues. J Dent Educ 66: 564-574, 2002

2. Sherr CJ: Cancer cell cycles. Science 274: 1672-1677, 1996.

3. Akervall JA, Michalides RJ, Mineta $\mathrm{H}$, et al: Amplification of cyclin D1 in squamous cell carcinoma of the head and neck and the prognostic value of chromosomal abnormalities and cyclin D1 overexpression. Cancer 79: 380-389, 1997.

4. Izzo JG, Papadimitrakopoulou VA, Li XQ, et al: Dysregulated cyclin D1 expression early in head and neck tumorigenesis: in vivo evidence for an association with subsequent gene amplification. Oncogene 17: 2313-2322, 1998.

5. Nakagawa $\mathrm{H}$, Wang TC, Zukerberg L, et al: The targeting of the cyclin D1 oncogene by an Epstein-Barr virus promoter in transgenic mice causes dysplasia in the tongue, esophagus and forestomach. Oncogene 14: 1185-1190, 1997.

6. Wilkey JF, Buchberger G, Saucier K, et al: Cyclin D1 overexpression increases susceptibility to 4-nitroquinoline-1-oxideinduced dysplasia and neoplasia in murine squamous oral epithelium. Mol Carcinog 48: 853-861, 2009.

7. Nishi K, Inoue H, Schnier JB and Rice RH: Cyclin D1 downregulation is important for permanent cell cycle exit and initiation of differentiation induced by anchorage-deprivation in human keratinocytes. J Cell Biochem 106: 63-72, 2009.

8. Ericson KK, Krull D, Slomiany P and Grossel MJ: Expression of cyclin-dependent kinase 6 , but not cyclin-dependent kinase 4 , alters morphology of cultured mouse astrocytes. Mol Cancer Res 1: 654-664, 2003.

9. Slomiany P, Baker T, Elliott ER and Grossel MJ: Changes in motility, gene expression and actin dynamics: Cdk6-induced cytoskeletal changes associated with differentiation in mouse astrocytes. J Cell Biochem 99: 635-646, 2006.

10. Matushansky I, Radparvar F and Skoultchi AI: CDK6 blocks differentiation: coupling cell proliferation to the block to differentiation in leukemic cells. Oncogene 22: 4143-4149, 2003.

11. Ogasawara T, Katagiri M, Yamamoto A, et al: Osteoclast differentiation by RANKL requires NF-kappa B-mediated downregulation of cyclin-dependent kinase 6 (Cdk6). J Bone Miner Res 19: 1128-1136, 2004.

12. Ogasawara T, Kawaguchi H, Jinno S, et al: Bone morphogenetic protein 2-induced osteoblast differentiation requires Smadmediated down-regulation of Cdk6. Mol Cell Biol 24: 6560-6568, 2004.

13. Dickson MA, Hahn WC, Ino Y, et al: Human keratinocytes that express hTERT and also bypass a p16(INK4a)-enforced mechanism that limits life span become immortal yet retain normal growth and differentiation characteristics. Mol Cell Biol 20: 1436-1447, 2000.

14. Van den Heuvel S and Harlow E: Distinct roles for cyclindependent kinases in cell cycle control. Science 262: 2050-2054, 1993.

15. Stewart SA, Dykxhoorn DM, Palliser D, et al: Lentivirusdelivered stable gene silencing by RNAi in primary cells. RNA 9: 493-501, 2003.

16. Coleman JE, Huentelman MJ, Kasparov S, et al: Efficient largescale production and concentration of HIV-1-based lentiviral vectors for use in vivo. Physiol Genomics 12: 221-228, 2003.

17. Bikle DD, Oda Y and Xie Z: Calcium and 1,25(OH)2D: interacting drivers of epidermal differentiation. J Steroid Biochem Mol Biol 89-90: 355-360, 2004

18. Hanahan D and Weinberg RA: The hallmarks of cancer. Cell 100: 57-70, 2000

19. Burnworth B, Popp S, Stark HJ, et al: Gain of 11q/cyclin D1 overexpression is an essential early step in skin cancer development and causes abnormal tissue organization and differentiation. Oncogene 25: 4399-4412, 2006.

20. Yamamoto H, Ochiya T, Takeshita F, et al: Enhanced skin carcinogenesis in cyclin D1-conditional transgenic mice: cyclin D1 alters keratinocyte response to calcium-induced terminal differentiation. Cancer Res 62: 1641-1647, 2002.

21. Bernards R: CDK-independent activities of D type cyclins. Biochim Biophys Acta 1424: M17-M22, 1999.

22. Ewen ME and Lamb J: The activities of cyclin D1 that drive tumorigenesis. Trends Mol Med 10: 158-162, 2004. 